\title{
Eficácia da ventosaterapia na dor lombar em vendedores do comércio no município de Santarém
}

\author{
Effectiveness of ventosa therapy on low back pain in commerce sellers in the city of Santarém \\ Eficacia de la terapia de viento sobre el dolor de espalda en vendedores comerciales de la ciudad \\ de Santarém
}

Recebido: 05/12/2021 | Revisado: 09/12/2021 | Aceito: 11/12/2021 | Publicado: 19/12/2021

\author{
Camila Eugênia Martins Lima \\ ORCID: https://orcid.org/0000-0002-8959-5850 \\ Instituto Esperança de Ensino Superior, Brasil \\ E-mail: camimartinslima@gmail.com \\ Karine Moreira da Silva \\ ORCID: https://orcid.org/0000-0002-5331-472X \\ Instituto Esperança de Ensino Superior, Brasil \\ E-mail: karinemoreiraa56@gmail.com \\ Jorge Carlos Menezes Nascimento Junior \\ ORCID: https://orcid.org/0000-0003-2593-7804 \\ Instituto Esperança de Ensino Superior, Brasil \\ E-mail: jcmnj@hotmail.com
}

\begin{abstract}
Resumo
Objetivo: Temos como objetivo desta pesquisa determinar a prevalência da eficácia da técnica ventosaterapia na dor lombar de origem muscular, mais especificamente em vendedores do comércio Métodos: Trata-se de uma pesquisa de campo com aplicação de questionários para coleta de dados, aplicação do protocolo de ventosaterapia, onde foi aplicado o questionário de coleta de dados para vendedores de ambos os sexos com dor lombar, em seguida realizou a aplicação da Escala visual analógica (EVA) no início e logo após cada sessão avaliando o nível da dor do paciente, totalizando 4 sessões ao todo, com duração em cerca de 15 a 20 minutos cada sessão, tendo um intervalo de cinco a sete dias entre as sessões. Conclusão: Conclui-se que a técnica de ventosaterapia tem resultados significativos para o tratamento da dor lombar, apresentando resultados positivos principalmente ao final das 4 sessões. Contudo novos estudos são necessários para melhor evidenciar o potencial da técnica.
\end{abstract}

Palavras-chave: Medicina tradicional chinesa; Dor crônica; Dor lombar; Ventosaterapia; Fisioterapia.

\begin{abstract}
Objective: The aim of this research is to determine the prevalence of the effectiveness of the technique of suction therapy in low back pain of muscle origin, specifically in vendors of the trade Methods: This is a field research with application of questionnaires for data collection, application of the suction therapy protocol, where the data collection questionnaire was applied to salesmen of both sexes with low back pain, then performed the application of the Visual Analog Scale (VAS) at the beginning and just after each session evaluating the level of the patient's pain, totaling 4 sessions in all, lasting about 15 to 20 minutes each session, having an interval of five to seven days between sessions. Conclusion: We conclude that the technique of suction cup therapy has significant results for the treatment of low back pain, showing positive results mainly at the end of the 4 sessions. However, new studies are necessary to better evidence the potential of the technique.
\end{abstract}

Keywords: Traditional Chinese Medicine; Chronic Pain; Backache; Suction therapy; Physiotherapy.

\section{Resumen}

Objetivo: El objetivo de esta investigación es determinar la prevalencia de la eficacia de la técnica de la ventosaterapia en las lumbalgias de origen muscular, concretamente en los vendedores del gremio: Se trata de una investigación de campo con aplicación de cuestionarios para la recogida de datos, aplicación del protocolo de ventosaterapia, donde se aplicó el cuestionario de recogida de datos a vendedores de ambos sexos con lumbalgia, realizándose posteriormente la aplicación de la Escala Visual Analógica (EVA) al inicio y justo después de cada sesión evaluando el nivel de dolor del paciente, sumando 4 sesiones en total, con una duración de unos 15 a 20 minutos cada sesión, teniendo un intervalo de cinco a siete días entre sesiones. Conclusión: Se concluye que la técnica de ventosaterapia tiene resultados significativos para el tratamiento del dolor lumbar, mostrando resultados positivos principalmente al final de las 4 sesiones. Sin embargo, se necesitan nuevos estudios para demostrar mejor el potencial de la técnica.

Palabras clave: Medicina tradicional china; Dolor crónico; Lumbalgia; Terapia del viento; Fisioterapia. 


\section{Introdução}

Aproximadamente $70 \%$ a $80 \%$ da população terá dores nas costas em algum momento de suas vidas, a parte inferior das costas é o principal local de envolvimento. A lombalgia é uma disfunção musculoesquelética caracterizada por dor, desconforto ou rigidez na região entre o arco costal final até a prega glútea, podendo ser considerada crônica ao longo de 12 semanas. A falta de ajuste dos músculos extensores do tronco leva à diminuição do controle e estabilidade do tronco, o que é essencial na realização de tarefas e manutenção da postura (Tozim, 2018).

Nos últimos anos, a dor lombar aumentou muito, e a fisioterapia tem sido usada diretamente para aliviar essa condição. A dor lombar é uma síndrome comum e causa inaptidão fazendo o paciente ausentar-se de suas atividades. Durante a sobrevida, a prevalência da síndrome é de 60-85\%. (Filho et al., 2009). Moreno, et al (2021) relata ainda que as mulheres apresentam fatores determinantes estando ligados diretamente a fisiologia hormonal, sendo que o estrógeno, hormônio predominante, acentuam os processos degenerativos articulares, afetando as estruturas ósseas e cartilagem articular, causando uma resposta inflamatória, o que causa uma desordem global. Sendo assim o gênero feminino o mais afetado.

A dor lombar pode causar alterações na flexibilidade corporal, redução da amplitude e fadiga dos músculos vertebrais, o que pode causar dor, incapacidade e aumento dos custos sociais. Isso representa uma maior taxa de absenteísmo e uma redução significativa no desempenho no trabalho. Essa taxa elevada é atribuída ao aumento da expectativa de vida, que desencadeia o desgaste natural da coluna vertebral (Faria \& Faria 2013).

A dor lombar aguda geralmente está relacionada ao envolvimento de ligamentos, músculos e ou disco intervertebral e é caracterizada por dor de início súbito que dura menos de seis semanas. Na maioria dos casos, é autolimitado e dura em média de um a sete dias. Aproximadamente $90 \%$ dos pacientes se recuperaram espontaneamente, $60 \%$ dos pacientes se recuperaram em um mês e 30\% a 60\% dos pacientes podem apresentar recorrência da dor em um a dois anos. A dor lombar subaguda dura de seis a doze semanas. Neste caso, as funções normais podem ser restauradas em até três meses. A dor lombar crônica ocorre apenas em cerca de $8 \%$ dos casos, ao longo de 12 semanas, prejudica a produtividade e é mais difícil de resolver completamente. Em estudo realizado no Brasil, cerca de 76,7\% das pessoas que sofrem de lombalgia crônica apresentam alta intensidade, o que acaba prejudicando o desempenho das atividades laborais (Junior, Goldenfum \& Siena 2010).

A etiologia da dor é desconhecida devido aos seguintes fatores risco, entre eles o trabalho repetitivo, como: ação de empurrar e puxar, o trabalho com a postura em tarefas estáticas e sentadas com vibrações no corpo inteiro, agachamentos, o girar ou levantar objetos repetidamente muito pesado, especialmente o peso que vai além da capacidade dos trabalhadores (Briganó \& Macedo 2005).

Segundo Moura (2018) uma das terapêuticas da MTC indicada para redução da dor crônica é a ventosaterapia que foi descoberta pelos chineses e egípcios que começaram a usar a ventosa terapia que ainda hoje é usada. No início de seu uso, esse método era aplicado por meio de chifres e cabaças. Por décadas, ele foi aprimorado pela medicina tradicional chinesa e tem sido usado em várias ventosas. As ventosas podem ser feitas de acrílico, vidro, plástico e, às vezes, de bambu. Ventosa, ou terapia ventosa, tem sido usada historicamente em muitas culturas, mas é considerada como uma terapia antiga da China e outros Países asiáticos (Ge et al., 2017). Nos últimos anos, tem ganhado popularidade em todo o mundo devido à sua aplicação simples, os efeitos adversos parecem mínimos e acredita-se em sua eficácia para a redução e regeneração da dor dos tecidos (Markowski et. al., 2014). Para Oliveira (2018), existem duas formas de se realizar o vácuo que ocorre no interior da ventosa, podendo ser colocando pegando fogo dentro da ventosa ou utilizando uma bomba de sucção.

A sucção da ventosa pode promover a ativação da circulação sanguínea, promover a melhora da circulação local e aliviar dores nas articulações e músculos. Segundo a pesquisa de Amaro (2015), esse método pode melhorar a resistência do organismo ao remover impurezas do sangue e reduzir a dor (Resende et al., 2019).

Para Focks (2008), a ventosaterapia pode ser aplicada no ponto principal de dor e em qualquer área do sistema 
musculoesquelético ou apenas no ponto de gatilho. Esse pensamento é contrário ao de Chilari, para ele o tratamento da ventosa terapia deve ser realizado em uma área de pelo menos 1,5 a 2,0 cm da área lesionada (Campos, 2015).

E justamente para aprofundar nossos conhecimentos sobre este tema, esse trabalho buscou o levantamento de material elaborado com a aplicação de questionários e intervenção prática com aplicação de ventosaterapia nos colaboradores do comércio do município de Santarém, na busca de comprovação da eficácia da ventosaterapia na dor lombar.

\section{Metodologia}

A pesquisa apresenta uma abordagem quantitativa pois os resultados obtidos são expressos na forma de dados numéricos, usando técnicas estatísticas para classificá-los e analisá-los como porcentagem, média e desvio padrão. Teve classificação descritiva com o objetivo de observar, registrar e descrever os próprios fatos. Possui uma cronologia transversal quando a pesquisa tem um período definido. Tratou-se de uma pesquisa de campo que visa utilizar técnicas de observação e questionários para coletas de dados, segundo Fontelles (2009).

Após a aprovação do Comitê de Ética em Pesquisa (CEP) CAAE: 46263321.60000 .5168 da Universidade do Estado do Pará, esta pesquisa ocorreu nos meses de setembro e outubro de 2021. Foi realizada em um escritório que fica situado no Centro comercial no município de Santarém-PA. Composta por vendedores de roupas que trabalham há pelo menos um ano nesta área, e com faixa etária de 19 a 30 anos, que possuem dor lombar de origem muscular e que aceitaram fazer parte da pesquisa. Foram incluídos na pesquisa colaboradores maiores de idade, que trabalham com vendas de roupas no centro comercial há mais de um ano, que não tenham outro vínculo empregatício formal ou informal e que não pratiquem atividades estudantis em paralelo ao trabalho.

Foram convocados 30 vendedores de ambos os sexos com queixa de dores lombares. Após a aplicação de um questionário com os critérios de exclusão e inclusão onde apenas 22 se encaixaram nos critérios da pesquisa, realizamos as sessões em 3 passos, sendo aplicado a escala EVA para avaliar o nível da dor, logo em seguida foi realizada a assepsia do local e aplicação de um óleo para facilitar a sucção do copo de ventosa, que foi aplicada com uma sucção média seguindo o protocolo Lombalgia 1 (pontos BP14, BP15, E25, R17, VC7, VC8 e VC10) conforme descreve Augusto Campos no livro Ventosaterapia o resgate de uma antiga arte: Longevidade. Os copos permaneceram a princípio 15 minutos, porém assim que atingiram uma coloração arroxeada regular estes foram retirados precocemente para evitar a formação de bolhas, após a aplicação retirou se os copos e foi realizado uma nova limpeza do local e o paciente foi orientado a não pegar sol e não aplicar nenhuma substância química sobre o local, e por último sendo aplicada novamente a escala EVA para identificar o nível da dor logo após a aplicação da ventosaterapia. Ao todo foram realizadas 4 sessões sendo 1 sessão por semana cada uma com duração de 15 a 20 minutos em média. Realizamos os atendimentos das sessões em uma sala totalmente reservada, climatizada e localizada no centro de Santarém.

As análises foram realizadas utilizando o software GraphPad Prism versão 9.0. Foram realizados testes de normalidades, para definir o padrão de distribuição dos dados (paramétricos ou não paramétricos). Em seguida foram realizados testes de comparação intragrupo (One way ANOVA) e entre grupos (pré-pós sessão - teste de Turkey).

Os dados estão apresentados em formato de tabelas e gráficos e foi considerado um valor de $\mathrm{p}<0,05$ para diferenças estatisticamente significativas (intervalo de confiança de $95 \%$ ).

\section{Resultados e Discussão}

Segundo Ribeiro et al., (2019) a Ventosaterapia é uma técnica milenar da Medicina Tradicional Chinesa, que utiliza um vácuo que realiza pressão negativa na pele e assim apresenta propriedades terapêuticas. Possui diversas formas de aplicação 
e pode ser ajustada a outras técnicas. A ventosaterapia surge como um meio alternativo ao tratamento de dores lombares, de forma com que os pacientes diminuam o uso de remédios com diversos efeitos colaterais que reduzem um problema, mas acabam trazendo outros. Ou seja, a eficácia do tratamento não é condicionada somente à redução da dor, mas também na fuga de meios invasivos e demais tratamentos à base de remédios (Campos, 2015).

De acordo com Cunha (2011), é possível citar três tipos de ventosas: A seca, onde a aplicação se dá com a utilização de várias ventosas sobre a região a ser tratada, podendo apresentar hematomas cujas cores servem de referência para avaliar a qualidade sanguínea da região; a molhada, onde a aplicação resulta na retirada de uma pequena porção sanguínea; e a deslizante, onde a aplicação causa menos hematomas que as técnicas tradicionais. Esta última é a mais utilizada pelos profissionais.

Gouveia (2008) diz que por não ter uma causa específica, sendo originária de vários fatores, o tratamento através da ventosaterapia é voltado para o alívio das dores, não sendo incomum o surgimento de demais benefícios de acordo com a frequência do tratamento. Segundo Brito et al., (2021) a técnica é de fácil aplicabilidade, custo baixo e curta duração, que proporciona efeitos locais e sistêmicos de forma instantaneamente. O vácuo causado pela sucção possibilita alívio dos processos dolorosos, aumento da circulação sanguínea, relaxamento tecidual e sensação de bem-estar.

Dessa forma, a ventosa terapia surge como um meio alternativo indicado para redução da dor com a aplicação de ventosas. São utilizados “copos” de diferentes materiais nas áreas sensíveis à dor juntamente com aparelhos de calor ou vácuo, objetivando causar estímulos para trocar gases com as células e limpar o sangue. O procedimento de ventosaterapia é capaz de auxiliar na limpeza sanguínea, fazendo a eliminação de gases impróprios decorrentes da má circulação. A diferença de pressão entre os meios internos e externos é o causador dessa troca, que é considerada similar ao que acontece nos pulmões, onde a grosso modo, entra oxigênio e gás carbônico sai (Campos, 2015).

Nesse sentido, nas palavras de Oliveira et al., (2018), a ventosa nada mais é do que uma cúpula de vidro aplicada na superfície da pele, onde será realizada uma pressão negativa com a utilização de uma bomba aspirante capaz de retirar o ar da cúpula. Esse procedimento que causa sucção da pele é importante para relaxar a musculatura e liberar os tecidos miofasciais.

A análise comparativa entre a escala EVA nas 4 fases pré versus 4 fases pós sessão revelou que houve redução significativa da dor lombar (valor da média pré 3,5 vs pós 1,4; p<0,0001) (Figura 1). 
Figura 1 - Comparação da escala EVA entre as fases pré e pós sessão.

\section{Escala EVA - pré e pós}

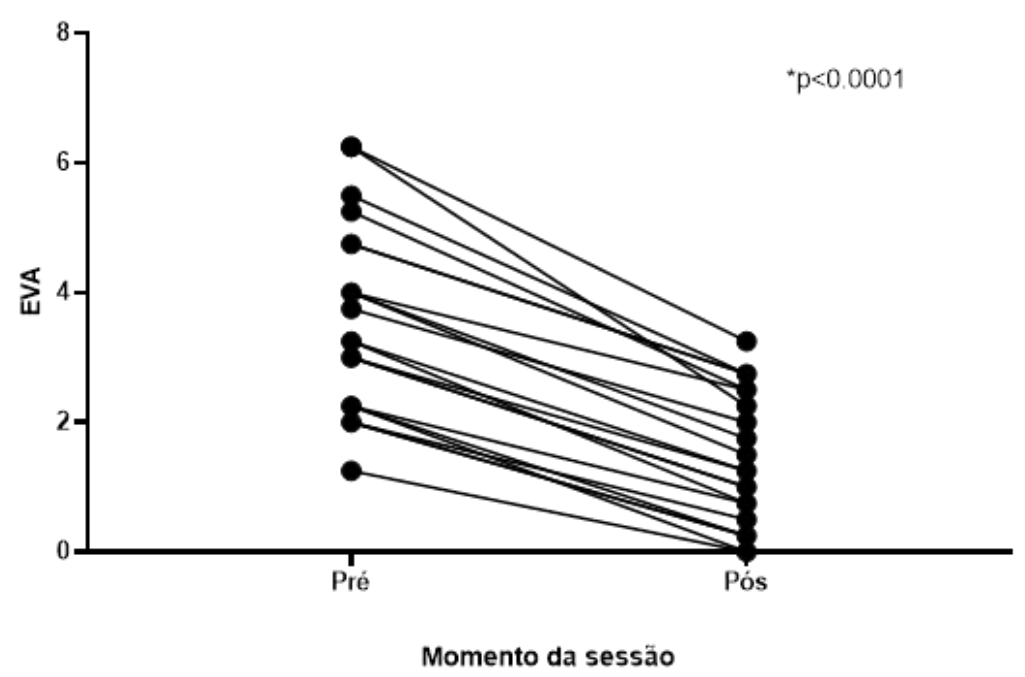

Fonte: Autores (2021).

De forma semelhante, a análise comparando todas as sessões, independente do momento pré ou pós, também demonstrou um padrão de redução da dor na escala EVA ao longo das sessões, conforme ilustrado no gráfico abaixo (Figura 2).

Figura 2 - Comparação da escala EVA entre todas as 2 fases das 4 sessões.

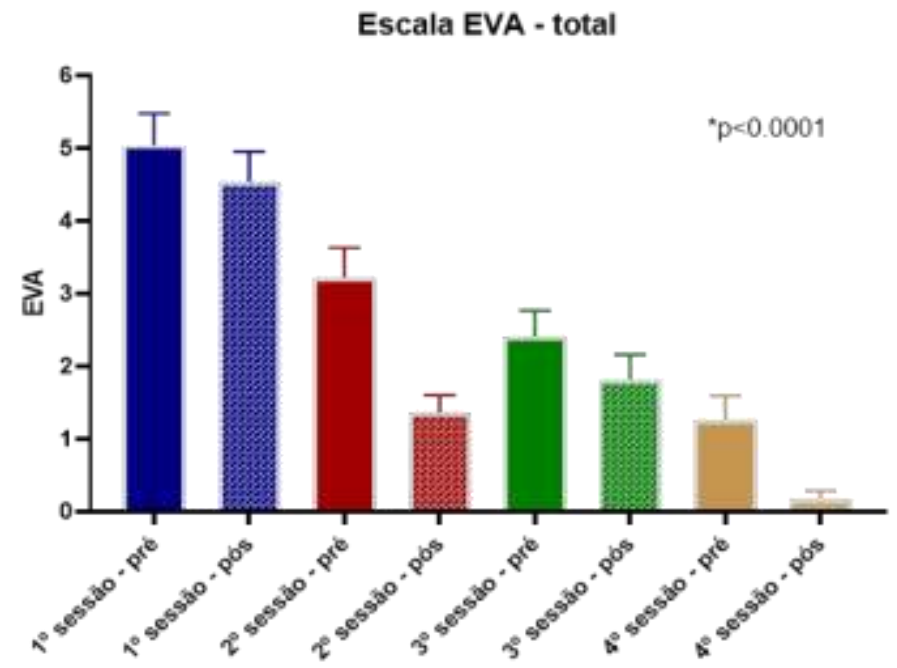

Fonte: Autores (2021).

Durante a análise de comparações múltiplas entre as sessões (Tabela 1), evidencia-se que não houve diferença estatisticamente significativa na escala EVA entre as fases pré e pós da primeira e da terceira sessão, ainda que houvesse tendência à redução. 
Tabela 1 - Comparações múltiplas da escala EVA entre as 2 fases (pré e pós) das 4 sessões.

\begin{tabular}{|c|c|c|c|}
\hline $\begin{array}{c}\text { Teste de Tukey } \\
\text { (múltiplas comparações) }\end{array}$ & $\begin{array}{l}\text { Diferença } \\
\text { média }\end{array}$ & IC $(95 \%)$ & Valor de p \\
\hline${ }^{\#} 1^{\circ}$ sessão - pré vs. $1^{\circ}$ sessão - pós & 0,5 & $-0,8195$ to 1,820 & 0,8996 \\
\hline $1^{\circ}$ sessão - pré vs. $2^{\circ}$ sessão - pré & 1,8 & 0,1721 to 3,464 & 0,0238 \\
\hline $1^{\circ}$ sessão - pré vs. $2^{\circ}$ sessão - pós & 3,7 & 2,332 to 5,031 & $<0,0001$ \\
\hline $1^{\circ}$ sessão - pré vs. $3^{\circ}$ sessão - pré & 2,6 & 2,030 to 3,243 & $<0,0001$ \\
\hline $1^{\circ}$ sessão - pré vs. $3^{\circ}$ sessão - pós & 3,2 & 2,125 to 4,329 & $<0,0001$ \\
\hline $1^{\circ}$ sessão - pré vs. $4^{\circ}$ sessão - pré & 3,8 & 2,137 to 5,408 & $<0,0001$ \\
\hline $1^{\circ}$ sessão - pré vs. $4^{\circ}$ sessão - pós & 4,9 & 3,412 to 6,316 & $<0,0001$ \\
\hline $1^{\circ}$ sessão - pós vs. $2^{\circ}$ sessão - pré & 1,3 & 0,1017 to 2,535 & 0,0277 \\
\hline $1^{\circ}$ sessão - pós vs. $2^{\circ}$ sessão - pós & 3,2 & 2,109 to 4,255 & $<0,0001$ \\
\hline $1^{\circ}$ sessão - pós vs. $3^{\circ}$ sessão - pré & 2,1 & 0,8253 to 3,447 & 0,0005 \\
\hline $1^{\circ}$ sessão - pós vs. $3^{\circ}$ sessão - pós & 2,7 & 2,023 to 3,431 & $<0,0001$ \\
\hline $1^{\circ}$ sessão - pós vs. $4^{\circ}$ sessão - pré & 3,3 & 2,081 to 4,465 & $<0,0001$ \\
\hline $1^{\circ}$ sessão - pós vs. $4^{\circ}$ sessão - pós & 4,4 & 3,124 to 5,603 & $<0,0001$ \\
\hline $2^{\circ}$ sessão - pré vs. $2^{\circ}$ sessão - pós & 1,9 & 0,9728 to 2,754 & $<0,0001$ \\
\hline${ }^{\#} 2^{\circ}$ sessão - pré vs. $3^{\circ}$ sessão - pré & 0,8 & $-0,6727$ to 2,309 & 0,6018 \\
\hline $2^{\circ}$ sessão - pré vs. $3^{\circ}$ sessão - pós & 1,4 & 0,09126 to 2,727 & 0,0307 \\
\hline $2^{\circ}$ sessão - pré vs. $4^{\circ}$ sessão - pré & 2,0 & 1,351 to 2,558 & $<0,0001$ \\
\hline $2^{\circ}$ sessão - pré vs. $4^{\circ}$ sessão - pós & 3,0 & 1,827 to 4,264 & $<0,0001$ \\
\hline${ }^{\#} 2^{\circ}$ sessão - pós vs. $3^{\circ}$ sessão - pré & $-1,0$ & $-2,181$ to 0,09011 & 0,0856 \\
\hline \# $2^{\circ}$ sessão - pós vs. $3^{\circ}$ sessão - pós & $-0,5$ & $-1,507$ to 0,5975 & 0,8244 \\
\hline${ }^{\#} 2^{\circ}$ sessão - pós vs. $4^{\circ}$ sessão - pré & 0,1 & $-0,6380$ to 0,8198 & 0,9999 \\
\hline $2^{\circ}$ sessão - pós vs. $4^{\circ}$ sessão - pós & 1,2 & 0,5720 to 1,792 & $<0,0001$ \\
\hline${ }^{\#} 3^{\circ}$ sessão - pré vs. $3^{\circ}$ sessão - pós & 0,6 & $-0,4122$ to 1,594 & 0,5192 \\
\hline${ }^{\#} 3^{\circ}$ sessão - pré vs. $4^{\circ}$ sessão - pré & 1,1 & $-0,2645$ to 2,537 & 0,1705 \\
\hline $3^{\circ}$ sessão - pré vs. $4^{\circ}$ sessão - pós & 2,2 & 1,040 to 3,414 & $<0,0001$ \\
\hline${ }^{\#} 3^{\circ}$ sessão - pós vs. $4^{\circ}$ sessão - pré & 0,5 & $-0,6578$ to 1,749 & 0,7885 \\
\hline $3^{\circ}$ sessão - pós vs. $4^{\circ}$ sessão - pós & 1,6 & 0,5210 to 2,752 & 0,0015 \\
\hline $4^{\circ}$ sessão - pré vs. $4^{\circ}$ sessão - pós & 1,1 & 0,2107 to 1,971 & 0,0088 \\
\hline
\end{tabular}

Fonte: Autores (2021).

Não houve diferença estatisticamente significativa $(p>0,05)$ na comparação pelo teste de Turkey; IC - intervalo de confiança. A partir dos dados apresentados, pode-se verificar a eficácia do protocolo da ventosa terapia para pacientes com lombalgia de origem muscular. Esses resultados possam servir de base para a formulação de planos de tratamento para pacientes com lombalgia e ampliar os métodos utilizados, além de promover discussões entre a população acadêmica e os profissionais de saúde, servir de referencial teórico e instrumentos de ensino-aprendizado.

\section{Conclusão}

A ventosaterapia é um método promissor para o tratamento e controle da dor lombar, uma vez que diminui significativamente as pontuações de intensidade de dor, em relação a um grupo ao controle. Em suma, a alta heterogeneidade e qualidade mediana metodológica dos ensaios clínicos randomizados limitou os resultados. Após o contato com os pacientes nas aulas práticas despertamos o interesse no tratamento da dor lombar, pois vimos que é uma condição que afeta muito os pacientes e nesse contexto identificamos ainda que os vendedores do comércio são pessoas que estão propensas a muitas dores, pois eles exercem atividades como levantar e baixar objetos, se inclinar, fazer arrumação de prateleira, além das vendas. Portanto, a maioria das pessoas se sente insatisfeita com as lombalgias, o que tem despertado a curiosidade sobre o assunto e o desejo de pesquisar sobre tal área tão recorrente e que afeta cada vez mais pessoas.

De acordo com esse estudo verificou-se que a técnica de ventosaterapia é um meio de intervenção eficaz para o tratamento da lombalgia de origem muscular, constatando que esta técnica apresentou resultados satisfatórios. A pesquisa mostrou que teve resultados positivos principalmente ao final das 4 sessões. No entanto, pela pouca quantidade de estudos 
sobre o efeito da ventosaterapia se faz necessário mais estudos relacionados a esta técnica milenar para que se possa determinar a efetividade da técnica.

\section{Referências}

Amaro, P. E. Q. (2015). Ventosaterapia no Tratamento de Acne Vulgar. Trabalho de Conclusão de Curso (Graduação Curso de Biomedicina) - Universidade Católica de Brasília.

Briganó, J. U. \& Macedo, C. S. G. (2005). Análise da mobilidade lombar e influência da terapia manual e cinesioterapia na lombalgia. Seminário: Ciências Biológicas e da Saúde, Londrina, 26(2), 75-82.

Brito, S. F. L., Salazar, A. S., Teixeira, M. E. M., Pinto, V. C. O., Silva, E. L. R., Rocha, V. A., Silva, M. G., Gonzaga, B G. L., Teixeira, F. T. A., Silva, M. A., Feitosa, M. C. P., Rêbelo, V. C. N., Neta, N. B. D., Carvalho, G. D. \& Carvalho, A. F. M. (2021). Análise dos efeitos da auriculoterapia e ventosaterapia no desempenho laboral de colaboradores de uma instituição de ensino privada em Teresina-PI. Research, Society and Development , $10(10)$, e 33101017951.

Campos, E. A. C. (2015). Ventosaterapia o resgate de uma antiga arte: longevidade. Ed. Andreoli.

Campos, G. H. \& Santos C. T. (2015). Tratamento de pontos-gatilhos (trigger points) por meio de terapia por ventosa. Revista de Divulgação Científica Sena Aires. 4(2), 146-154.

Cunha, A. A. (2011). Ventosaterapia Tratamento e Prática. (3a ed.) Ed. Icone.

Faria, M. B. M. \& Faria, W. C. (2013). The effect of the method pilates in the treatment of pain lumbar chronic nonspecific - a review of the literature. Revista Científica do UNIFOR-MG. 8(1), 75-84.

Filho, J. L. B. \& Braun, L. M. (2009). Radiofrequency in chronic pain. Rev. Coluna/Columna. 8(2), 200-205.

Focks, C. \& Mark, U. (2008). Guia Prático de Acupuntura: localização de pontos e técnicas de punção, Ed. Barueri.

Fontelles, Mauro, J., Simões, M. G., Farias, S. H. \& Fontelles, R. G. S. (2009). Metodologia da pesquisa científica: diretrizes para a elaboração de um protocolo de pesquisa. Núcleo de Bioestatística Aplicado à Pesquisa da Universidade da Amazônia - Unama. Amazonas.

Ge, W., Leson, C. \& Vukovic, C. (2017). Dry cupping for plantar fasciitis: a randomized controlled trial. Rev. J Phys Ther Sci. 29(5), 859-862.

Gouveia, K. M. C. \& Gouveia, E. C. (2008). O músculo transverso abdominal e sua função de estabilização da coluna lombar. Rev. Fisioterapia movimento. 21(3), 45-50.

Junior, M. H., Goldenfum, M. A. \& Siena, C. (2010). Occupational low back pain. Revista da Associação Médica Brasileira. 56(5), 583-589.

Markowski, A., Sanford, S., Pikowski, J., Fauvell, D., Cimino, D. \& Caplan, S. (2014). Um estudo piloto que analisa os efeitos da ventosa chinesa como um tratamento adjunto para pacientes com dor lombar subaguda no alívio da dor, na melhoria da amplitude de movimento e na melhoria da função. Rev. The Journal of Alternative and Complementary Medicine. 20(2), 113-117.

Moreno, A. G. U. T., Bezerra, A. G. V., Silva, E. G. A., Melo, E. L., Gerbi, M. E. M. M., Bispo, M. E. A., SÁ, R. A. G. \& Menezes, M. R. A. (2021). Influência do estrógeno na modulação da dor na disfunção temporomandibular e sua prevalência no sexo feminino: revisão integrativa. Research, Society and Development. 10(2), e38510212453

Oliveira, M. A. R., Silva, A. P. \& Pereira L. P. (2018). Ventosaterapia - Revisão De Literatura. Revista Saúde em Foco. 10 ed. $151-154$.

Resende, G. O., Melo, C. M., Silva, G T., Nunes, I. S., Rossi, J. V., Angelim L. S., Perreira, S. L. S. \& Nishi, M. (2019). Ventosaterapia e Acupuntura no Tratamento De Lombalgia, Anais da XVII Mostra Acadêmica do Curso de Fisioterapia. 7(2), 22-29.

Ribeiro, J. C., Sêne, R. F., Paiva, A. F. A., Pinto, L. P. \& Assis I. B. (2019). Ventosaterapia: tratamento alternativo para diversas afecções. Revista Saúde em Foco. (11a ed.), 1381-1393.

Tozim, B. M. (2018). Efeito do método pilates nos parâmetros de desempenho neuromuscular em idosos com dor lombar. Tese de doutorado, Instituto de Biociências (Pós-graduação Desenvolvimento Humano e Tecnologias - IBRC) - Rio Claro.

Moura, C. de C., Chaves, E. de C. L., Cardoso, A. C. L. R., Nogueira, D. A., Corrêa, H. P. \& Chianca, T. C. (2018). Ventosaterapia e dor crônica nas costas: revisão sistemática e metanálise. Rev. Latino-Am. Enfermagem. 26, e3094. 